\title{
COMPARISON OF MICROFOCUS- AND SYNCHROTRON X-RAY TOMOGRAPHY FOR THE ANALYSIS OF OSTEOINTEGRATION AROUND TI6AL4V-IMPLANTS
}

\author{
R. Bernhardt ${ }^{1}$, D. Scharnweber ${ }^{1}$, B. Müller ${ }^{2 *}$, P. Thurner ${ }^{3}$, H. Schliephake ${ }^{4}$, P. Wyss ${ }^{3}$, F. Beckmann ${ }^{5}$, \\ J. Goebbels ${ }^{6}$, and H. Worch ${ }^{1}$ \\ ${ }^{1}$ Max-Bergmann-Center of Biomaterials, TU Dresden, D-01062 Dresden, Germany, \\ ${ }^{2}$ Computer Vision Laboratory, Swiss Federal Institute of Technology, CH-8092 Zürich, Switzerland \\ ${ }^{3}$ Swiss Federal Laboratories for Materials Testing and Research, CH-8600 Dübendorf, Switzerland \\ ${ }^{4}$ Department of Cranio-Maxillofacial Surgery, Georgia-Augusta-University, D-37075 Göttingen, Germany \\ ${ }^{5}$ GKSS-Research Center, D-21502 Geesthacht, Germany \\ ${ }^{6}$ Federal Institute for Materials Research and Testing, D-12200 Berlin, Germany
}

\begin{abstract}
Micro-computed tomography $(\mu \mathrm{CT})$ provides quantitative three-dimensional information of bone around titanium implants similar to classical histology. The study, based on an animal model, using cuboid-shaped biofunctionalised Ti6Al4V implants with surrounding bone after 4 weeks, is performed using $3 \mu \mathrm{CT}$-systems with X-ray tubes, one synchrotron-radiation-based $\mu \mathrm{CT}$-system $(\mathrm{SR} \mu \mathrm{CT})$, and classical histology. Although the spatial resolution of the $\mu \mathrm{CT}$ systems is comparable, only the results of SR $\mu \mathrm{CT}$ agree with results of classical histology. The X-ray tube sources give rise to huge artefacts in the tomograms (interface scattering, beam hardening), which impaired the quantitative analysis of bone up to about $200 \mu \mathrm{m}$ from the implant surface. Due to the non-destructive character of $\mu \mathrm{CT}$ the specimens can be subsequently examined by classical histology without restriction. The quantitative comparison of bone formation uncovers the strong dependence of the detected amount of newly formed bone from the selected slice. This implies the necessity of 3D analysis. SR $\mu \mathrm{CT}$ and classical histology prove that surface modifications of the titanium implant significantly influence the bone formation. Using $\mathrm{SR} \mu \mathrm{CT}$, the preparation artefacts due to cutting and polishing are excluded.
\end{abstract}

Key Words: X-ray micro computed tomography, comparison of microfocus and synchrotron radiation, osteointegration, surface modification of titanium implants, histology, bone segmentation, quantification of bone formation

*Address for correspondence:

B. Müller

Computer Vision Laboratory,

Swiss Federal Institute of Technology,

CH-8092 Zürich,

Switzerland

Fax Number: +41 16321199

bmueller@vision.ee.ethz.ch

\section{Introduction}

A main goal of today's biomaterials research for mechanically loaded bone implants is the improvement of the osteoconductivity and/or osteoinductivity of the implant surfaces (LeGeros and Craig, 1993; Johnsson et al., 2000; Truhlar et al., 2000; Giavaresi et al., 2003). Concerning the generation of defined surface morphologies and inorganic coatings using calcium phosphate phases, recent research activities concentrate on the incorporation of organic components (peptides, extracellular matrix proteins, growth factors) into the biomimetic design of surface layers (Dard et al., 2000; Becker et al., 2002; Schliephake et al., 2003).

Conventional histology is a well-established technique to characterise the biological reaction to surface-modified implants (Chappard et al., 1999). Depending on the microscopic equipment the lateral resolution reaches the sub-micrometer level (Turner et al., 1989; Fartash et al., 1990; Wennerberg et al., 1995; Hallgren et al., 2001; Ivanoff et al., 2001). The samples, however, have to be cut into slices with the thickness of at least $50 \mu \mathrm{m}$, which are usually polished. These procedures often result in artefacts at the tissue-implant interface, which are more pronounced if the mechanical properties between tissue and implant differ strongly, such as for ceramics and metals. Due to losses during the preparation, the vertical resolution of histological investigations of mineralised bone around implants is approximately $250 \mu \mathrm{m}$ (Wolf and Pompe, 1980).

Micro-computed tomography $(\mu \mathrm{CT})$, a non-destructive technique, can provide a spatial representation of bone formation at the implant surface and the peri-implant region up to a few micrometers or even better. As the result virtual cuts with identical resolution in any direction can be generated from $\mu \mathrm{CT}$ tomograms.

For the quantification of bone (density or trabecular structure), $\mu \mathrm{CT}$ has been shown to be a very powerful technique (Morton et al., 1990; Ruegsegger et al., 1996; Kron, 1998; Kinney et al., 2000; Dempster et al., 2001; Nuzzo et al., 2002). For example, quantitative micro-computed tomography with synchrotron radiation has been successfully used to investigate the 3D microstructure and the degree of bone mineralization at high spatial resolutions (voxel size of $10 \mu \mathrm{m}$ ) (Nuzzo et al., 2002). With regard to osteoporosis an increase of connectivity density was found by $\mu \mathrm{CT}$-measurements of bone biopsy 
Table 1. Experimental parameters for the $\mu \mathrm{CT}$-measurements

\begin{tabular}{|c|c|c|c|c|}
\hline \multirow[b]{2}{*}{ experimental parameter } & \multicolumn{4}{|l|}{$\mu \mathrm{CT}$-systems } \\
\hline & HASYLAB at DESY & BAM & EMPA & TU Dresden \\
\hline X-ray source & $\begin{array}{l}\text { synchrotron } \\
\text { radiation }\end{array}$ & $\begin{array}{l}\mu \text {-focus tube } \\
\text { (Feinfokus, } \\
\text { Germany) }\end{array}$ & $\begin{array}{l}\mu \text {-focus tube } \\
\text { (Feinfokus, } \\
\text { Germany) }\end{array}$ & $\begin{array}{l}\mu \text {-focus tube } \\
\text { (MediXtec, } \\
\text { Germany) }\end{array}$ \\
\hline X-ray energy & $=60 \mathrm{keV}$ & $<100 \mathrm{keV}$ & $<142 \mathrm{keV}$ & $<100 \mathrm{keV}$ \\
\hline Magnification & 2.6 & 6 & 6 & 15 \\
\hline Reconstructed voxel size & $6.4 \mu \mathrm{m}$ & $9.7 \mu \mathrm{m}$ & $18 \mu \mathrm{m}$ & $20 \mu \mathrm{m}$ \\
\hline Reconstructed image size & $1274 \times 1274$ & $921 \times 921$ & $1024 \times 1024$ & $512 \times 512$ \\
\hline Filter & monochromator & $0.2 \mathrm{~mm} \mathrm{Cu}$ & $0.05 \mathrm{~mm} \mathrm{Ag}$ & none \\
\hline Radiographs & 720 & 720 & 600 & 360 \\
\hline
\end{tabular}

specimens (Dempster et al., 2001). The number of communications related to bone quantification around implants with $\mu \mathrm{CT}$, however, is rather small (Wigianto et al., 1999; Van Oosterwyck et al., 2000; Sennerby et al., 2001), and, so far, quantitative studies on osteoinductivity and osteointegration of metal implants using synchrotron radiation (SR) are unavailable.

Titanium, which is a widely used material for load bearing implants, exhibits much stronger X-ray absorption than bone. During the first stages of bone formation the difference in absorption is especially high since the tissue is not fully mineralised and thus mainly composed of light elements. Consequently, the visualization of the interface is complicated and a quantitative evaluation becomes difficult, as already shown by $\mu \mathrm{CT}$ using microfocus X-ray sources (Van Oosterwyck et al., 2000; Sennerby et al., 2001).

To compare the advantages and limitations of microfocus-CT and SR $\mu \mathrm{CT}$ characterizing bone formation near titanium implants, we have performed a comprehensive study of a series of explants using different $\mu \mathrm{CT}$-systems.

Through the use of the animal model we have compared the results of bone formation around titanium implants from common histological images to the $\mu \mathrm{CT}$ tomograms using semi-automated image processing for segmentation and bone quantification.

\section{Sample preparation}

\section{Materials and Methods}

For the experimental study, cuboid shaped Ti6Al4V implants with an oblique diameter of $4.6 \mathrm{~mm}$ and $13 \mathrm{~mm}$ in length were polished and modified with the following methods: anodic oxidation (Scharnweber et al., 2002), collagen partially integrated in the oxide layer (Scharnweber et al., 2004), RGD-peptide immobilization (Roessler et al., 2001), coating with hydroxyapatite (Roessler et al., 2003), coating with mineralised collagen (Dard et al., 2000). According to an experimental model (Becker et al., 1991) the implants were inserted into trephine burr holes of $4.6 \mathrm{~mm}$ in the lower jaw of beagle dogs by press-fitting (Schliephake et al., 2002). After 4 weeks healing time mandibular segments were retrieved and immediately fixed in $4 \%$ buffered formalin. The individual implants with surrounding bone were embedded into poly-methylmethacrylate (PMMA). For the $\mu \mathrm{CT}$-examinations 6 cylindrical specimens with a diameter of $8 \mathrm{~mm}$ were examined prior to the histological investigation. The implant long axis was nearly centred in the bone-embedding compound.

\section{Micro-computed tomography experiments}

Besides the microfocus-CT-systems at the Federal Institute for Materials Research and Testing, Berlin (BAM) (Goebbels et al., 2002), the Swiss Federal Laboratories for Materials Testing and Research, Dübendorf, Switzerland (EMPA) and Dresden University of Technology, Germany (TUD) (Nöthe et al., 2002), we used a SR $\mu$ CT-system at the Hamburger Synchrotronstrahlungslabor of the Deutsches Elektronen-Synchrotron (HASYLAB at DESY, high energy beam-line BW5). The experimental parameters for $\mu \mathrm{CT}$ in absorption contrast mode are given in Table 1. The selection of optimized experimental parameters for the conventional $\mu \mathrm{CT}$-systems (photon energy, voxel size, incorporation of appropriate filters, and magnification) depends on the specific components the systems are made of.

The BAM detector system consists of a cooled CCDcamera (Photometrix, Melbourne, Australia) (Series 200, CCD-type TK1024 AF2 with $1024 \times 1024$ pixels, pixel length $24 \mu \mathrm{m}$ ) coupled to a 2:1 reducing fiber taper, which is coated with a GdOS scintillator screen about $25 \mu \mathrm{m}$ thick. The read-out rate of the 16-bit CCD-camera corresponds to $200 \mathrm{kHz}$. The $0.2 \mathrm{~mm}$-thick $\mathrm{Cu}$-filter was used to reduce the relative amount of low-energy photons and thus improves the X-ray spectrum with respect to the expected beam hardening effects.

The detection unit for the EMPA system consists of a $6.35 \mathrm{~mm}$-thick scintillating fiberoptic faceplate of type LKH6 (Collimated Holes Inc, Campbell, CA, USA) combined with the 12-bit CCD-camera MCD1200 (Spectrasource Instruments, Westlake Village, CA, USA) with the resolution of $1024 \times 1024$ pixels. Using the accelerating voltage of $142 \mathrm{keV}$, the choice of the $0.05 \mathrm{~mm}$ thick Ag-filter leads to the significant reduction of the lowenergy photons without substantially diminishing the intensity of higher energetic photons. In order to increase the density resolution, the binning mode of the camera (binning factor 2) has been used. 


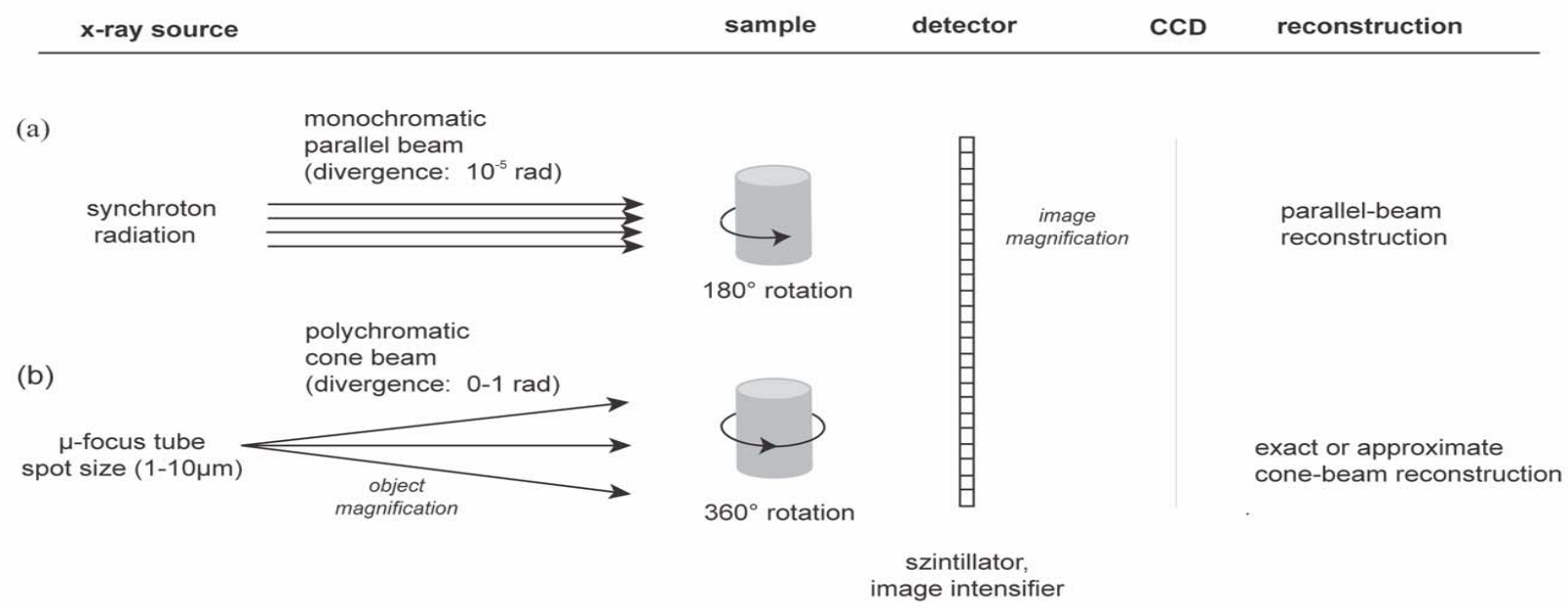

Figure 1. The schematic drawings show the similarities and the differences between (a) SR $\mu \mathrm{CT}$ with monochromatic parallel X-rays and (b) microfocus- $\mu \mathrm{CT}$ using the polychromatic cone beam.

The detection unit of the TUD-system comprises the image intensifier (Thomson TH9430) (Thomson Tube Electronics, now: Thales Electron Devices, Vélizy Cedex, France) combined with the 12-bit CCD-camera CH350 (Photometrix) with 1024x1024 pixels. For the TUD-system, no filter was applied because of the large sample-detector distance chosen, resulting in a relatively low photon density at the sample position.

Characteristic for the synchrotron radiation based setup (cf. Fig. 1a) is the parallel monochromatic X-ray. Using the double bent Laue monochromator made of Si(111) the photon energy becomes tuneable and thus adjustable to the thickness and composition of the sample (Beckmann et al., 1997; Beckmann et al., 2000). Behind the sample, the single crystal $\left(\mathrm{CdWO}_{4}\right)$ fluorescence screen transforms the attenuated $\mathrm{X}$-rays into visible light that is magnified to a 12-bit CCDcamera (KX2 by Apogee Instruments Inc, San Jose, CA, USA). The reconstruction of the radiographs was done with the filtered back-projection algorithm (Kak and Slaney, 2001).

The main difference of microfocus-CT systems in relation to $\mathrm{SR} \mu \mathrm{CT}$ is the type of the X-ray source. The use of Xray tubes leads to relatively low intense, conical beams with the continuous white energy spectrum, as schematically shown in Figure 1b. Metallic filters between tube and sample reduce the beam hardening effect. Due to the conical beam propagation the magnification is adjustable and related to the position of the sample between source and detector. The detector including CCD-camera is similar to the one of SR $\mu \mathrm{CT}$-systems. Radiographs obtained from microfocus-CT devices were reconstructed with a Feldkamptype cone-beam reconstruction algorithm (Feldkamp et al., 1984).

\section{Histological investigation}

For the histological investigation non-decalcified sections of the samples with a thickness of 30 to $70 \mu \mathrm{m}$ perpendicular through their long axis were prepared. The vertical resolution was about $1 \mathrm{~mm}$. The resulting slices were surface stained with alizarin-methylene-blue and von Kossa stain. To view the whole tissue around the implants from each slice, micrographs with an image size of $900 \times 600$ pixels (pixel size: $12.6 \mu \mathrm{m}$ ) were taken.

\section{Quantification of osteointegration}

To segment the bony structures in the region of interest (ROI) in the histological images and the $\mu \mathrm{CT}$-tomogram, different procedures were used. In the classical histological images, the segmentation of pixels related to bony tissue was done with a colour splitting procedure and histogram arrangements. If the threshold for the visible bony structure could not be found automatically, the segmentation was done manually. Here, characteristic bony features such as lacunae and osteocytes were selected. Areas with titanium particles, which presumably originate from the cutting process, were discarded. The extraction of bone-related absorption values in the $\mu \mathrm{CT}$ tomograms was based on absorption histograms obtained by image analysis software (ImageJ, National Institutes of Health, USA; VGStudioMax 1.1, Volume Graphics $\mathrm{GmbH}$, Heidelberg, Germany). Due to the intensitybased binary conversion of the grey-level distributions with the threshold, the components in the tomograms were separated. The noise is further reduced with a structural image filter (ImageJ, noise/despeckle) retaining the overall bony structure. SR $\mu \mathrm{CT}$ tomograms from the HASYLAB at DESY lead to histograms of the local absorption coefficients that have exactly the Gaussian shape (Müller et al., 2002). Therefore, it is rather simple to determine the threshold for the segmentation of different components such as bone and embedding material. The tomograms obtained with the conventional X-ray sources, however, provide histograms that exhibit various deviations from the Gaussian shape. In order to demonstrate the phenomenon in a semi-quantitative way we have fitted the part of the histogram, which is related to embedding and bone, with two Gaussians for the four $\mu \mathrm{CT}$-systems by the use of the Levenberg-Marquardt al- 

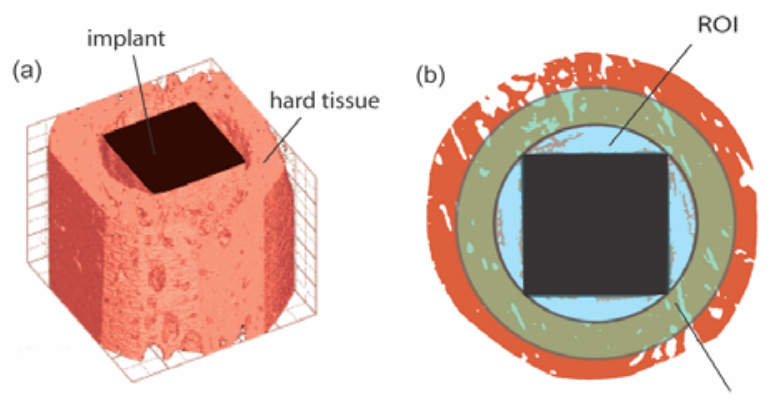

(c)

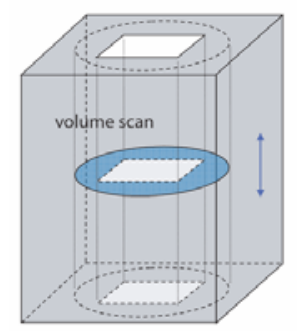

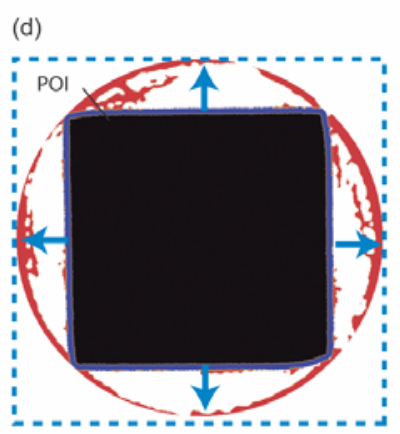

Figure 2. In (a) the sample geometry is demonstrated using the 3D-representation of the implant (black) with surrounding bony tissue (red). In (b) a typical slice is selected to show the cylindrical region of interest (ROI) blue-coloured around the implant (black) and the reference area (green-coloured). (c) The scheme shows the integration of the ROI along the implant symmetry axis in the volumetric $\mu \mathrm{CT}$-data set. In (d) the procedure that defines the perimeter of interest (POI), which is pixelwise expanded from the bone/implant interface to the outer diameter of the ROI, is given.

gorithm (Marquardt, 1969) of the ProFit code (profit 5.5.3, Quantumsoft, Zürich, Switzerland).

To determine the amount of newly formed bone, the cylindrical hole for the implant in the jaw-bone was selected as the ROI, as shown in Figure 2b. As the implant axis in the samples is not fully adjusted to the z-axis of the $\mu \mathrm{CT}$ tomogram, a spatial reorientation of the longitudinal implant axis was done to position the ROI for the automatic slice scanning (cf. Fig. 2c). The implant adjustment was carried out comparing the implant position at the top and the bottom slices to calculate the two transformation angles. The rotation of the $\mu \mathrm{CT}$ tomogram was done with an Image J plug-in (Transform J v2.1, ${ }^{\circ}$ Erik Meijering, Rotterdam, The Netherlands). For the detailed comparison of accumulated bone the tomograms were sliced as subsequently done by histology. Because of the different local bone structures, the reference area for each slice as shown in Figure $2 \mathrm{~b}$ was used, which corresponds to $1.5 \mathrm{x}$ ROIdiameter.

Due to the symmetric implant design, the binary slice stack containing the bony structure could be simply projected (z-project, ImageJ). The resulting image divided by the certain absorption values is the normalised slice along the implant axis, which gives rise to an image containing floating-point values between zero (absence of bone values) and one (bone values at each point along the implant).

Note that the implant is discarded in the resulting image. To quantify the peri-implant bone amount, analysis procedures (cf. Fig. 2d) were developed using IDL 5.4 (Research Systems, Inc., Boulder, CO, USA) and ImageJ as the programming languages. The titanium surface in the images, as the starting point in the peri-implant analysis, was automatically selected due to its strong contrast in relation to the values for bone and embedding material. A reliable method here is to start with the pixel-scan at any point of the implant and to continue the scan in $\mathrm{x}$ - and $\mathrm{y}$ directions until the peri-implant interface $(\mathrm{M})$ is reached. These points are defined as the perimeter of interest (POI, Fig. 2d). The division of accumulated pixel values for $M$ by the pixel number leads to a relative bone amount for the implant surface. To analyze changes in the bone quantity at a certain implant distance, the POI was expanded pixel-wise and the related perimeter values were determined, as shown in Figure 2d. The standard deviation (SD) of the mean bone value was calculated using a confidence interval of $95 \%$.
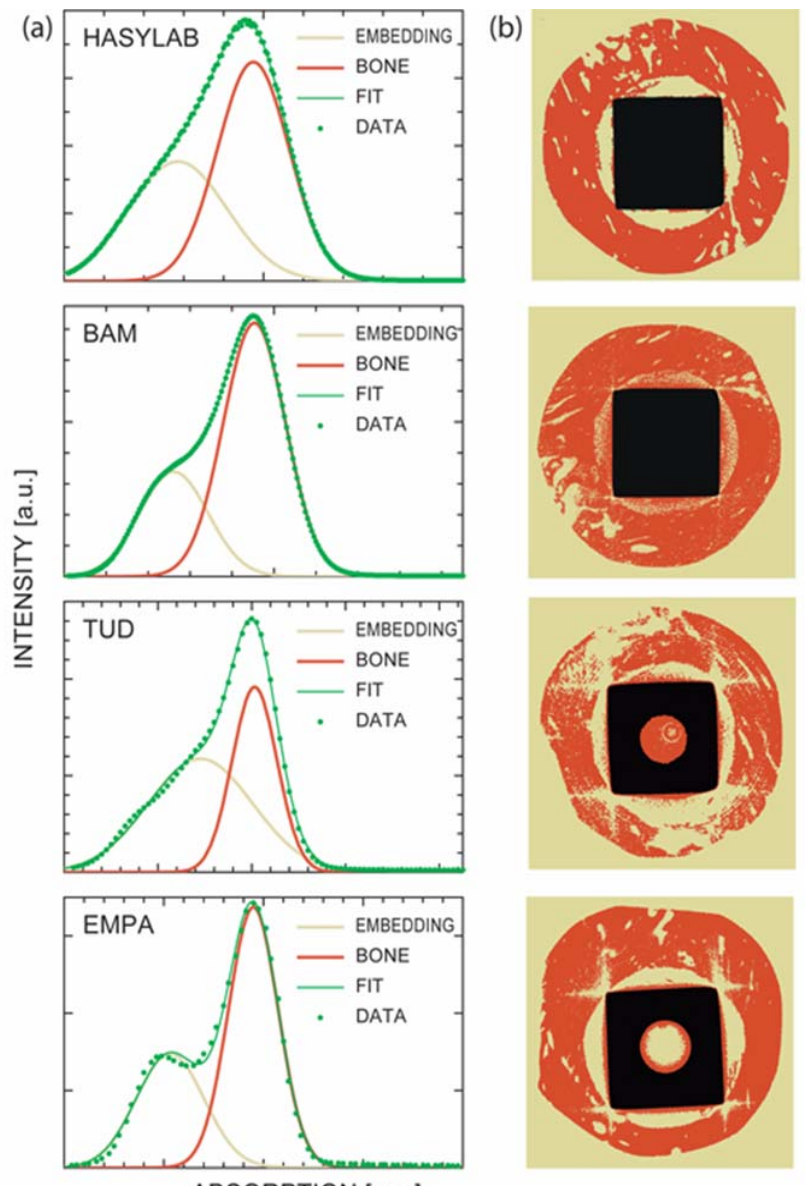

ABSORPTION [a.u.]

Figure 3. The histograms of the X-ray absorption for the tomograms of the different $\mu \mathrm{CT}$-systems (a) exhibit more or less the Gaussian shape. The related typical slices, which are thresholded using the crossing points of the Gaussians, make visible the mineralised bone around the implants (b) and demonstrate the artefacts as the result of interface scattering. 

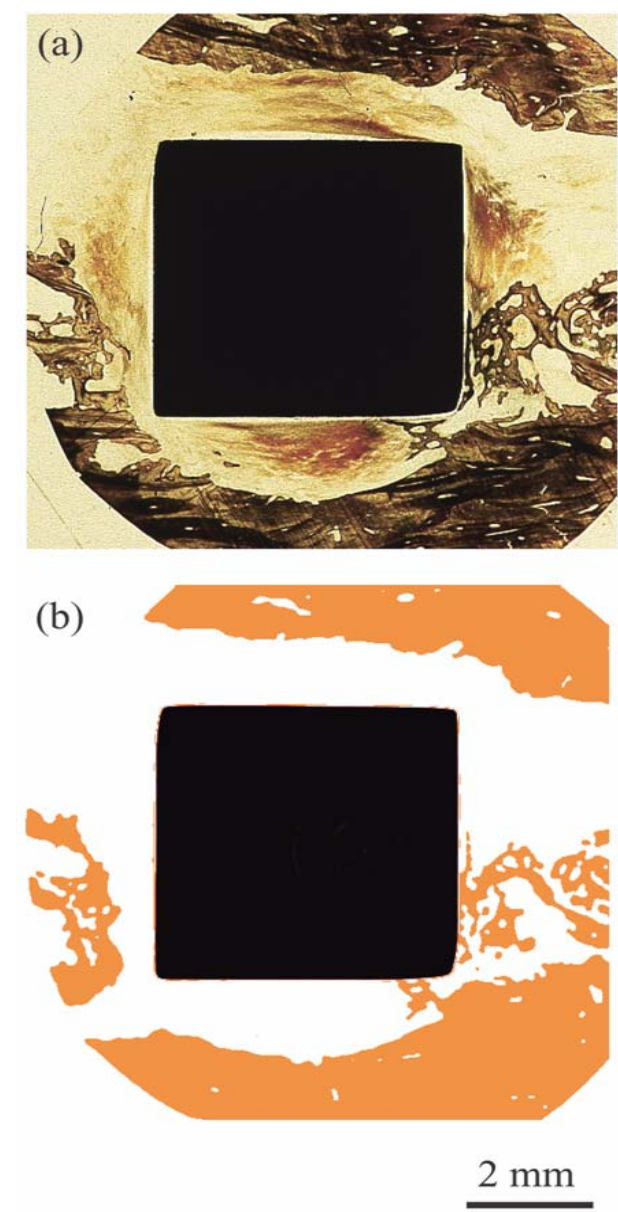

Figure 4. The micrograph of the cross section from classical histology is comparable with the related $\mathrm{SR} \mu \mathrm{CT}$ slice. The Ti6Al4V cuboid-shaped implant (black) is put into a cylindrical hole in the dogs jaw. The bony tissue is von Kossa stained. The dark colour of the diffuse mineralising fibrosis complicates the automatic quantitative evaluation inside the ROI. The absence of such an effect inside the ROI of the SR $\mu \mathrm{CT}$ images is essential for the automatic data analysis.

\section{Results \\ Bone thresholding and filtering}

The X-ray absorption histograms (Fig. 3a) obtained from $\mu \mathrm{CT}$-tomograms of the same sample geometry with different X-ray sources show an overlap of distributions for bone and embedding material. The threshold between titanium (not shown in the histograms) and tissue is obvious, due to a clear peak separation. With the Gaussianfitting algorithm, embedding and mineralised bone is segmented. To define threshold values between both components, the point of intersection between the fitted curves was chosen. Figure $3 \mathrm{~b}$ shows characteristic $\mu \mathrm{CT}$-slices using the fitted threshold for the different $\mu \mathrm{CT}$-systems. The thresholded slices from conventional $\mu \mathrm{CT}$-systems (BAM, TUD, EMPA) represent several artefacts at the implant corners and around the implant surface.

The overlap of the absorption values for bone and embedding material in the thresholded slices originates from the significant noise inside the ROI. The structural image filter (ImageJ, noise-despeckle) reduces the noise, retaining the overall bone structure, only for the tomogram ob-

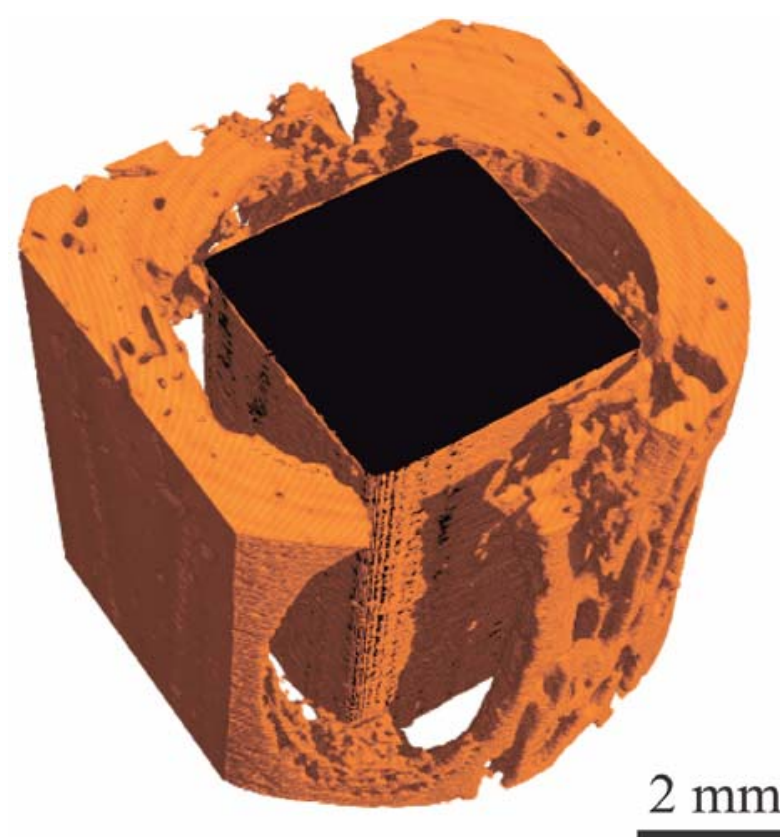

Figure 5. For the qualitative 3D-evaluation of the newly formed mineralised bone it is very helpful to generate the 3D-representation of the implant and the bone. Here, a set of $477 \mathrm{SR} \mu \mathrm{CT}$-slices, as shown in Figure 4, gives the spatial impression of bone around the implant. As expected, the osteointegration of the implant after 1 month healing time is mainly observed near the edges of the implant surface.

tained at HASYLAB (Fig. 3b). Figure 4 shows the good agreement of a thresholded and filtered SR $\mu \mathrm{CT}$-slice with the corresponding histological image for the bony tissue. Applying the rendering procedure (VG Studio) to the thresholded absorption values for bone, the $3 \mathrm{D}$ representation of bone morphology around the implant surface becomes clearly visible (Fig. 5).

The automatic bone selection in the histological images was rather difficult to obtain. It depends mainly on the staining procedure used as well as the quality of the preparation. If the alizarin-methylene-blue stain is used, which leads to an orange colour of mineralised bone, reasonable values for the threshold, which closely fit to the bone morphology, are found. The use of the von Kossa stain leads to brown coloured bony tissue. Here, it is hard to distinguish between the implant and the stain inside the ROI (Fig. 4a). In this case the bone was selected manually.

\section{Bone quantification}

According to the described procedures, Figure 6 shows the relative amount of newly formed bone inside the ROI integrated over the 5 histological images and over about $500 \mu \mathrm{CT}$-slices of the Sample 29, where the titanium was coated with RGD. There is no significant difference in the mean values for bone, which is $15.5( \pm 4.5) \%$ for the classical histology and $14.6( \pm 2.7) \%$ for the $\mathrm{SR} \mu \mathrm{CT}$-measurement. As mentioned above, the artefacts inside the ROI for the microfocus-CT investigations result in a prominent overestimation of bone, which is $20.1( \pm 7.8) \%$ for the BAM-system and $32.0( \pm 5.8) \%$ for the TUD-system. 


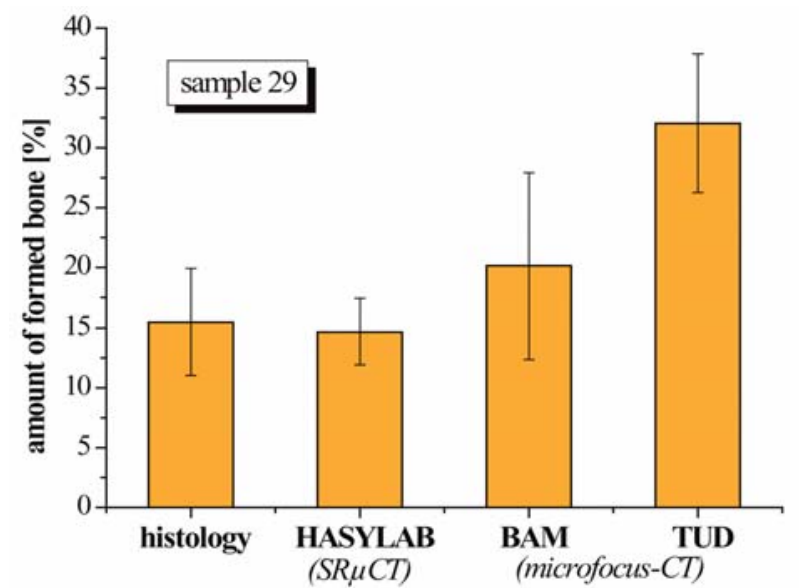

Figure 6. The amount of bone for Sample 29, which is formed in the region of interest, corresponds to about $15 \%$, as verified by classical histology and $\mathrm{SR} \mu \mathrm{CT}$. The limited number of slices in classical histology (5) results in the rather large error bar. The results from the microfocus- $\mu \mathrm{CT}$-systems overestimate the bone formation and give rise to significantly higher standard deviations, although the integration is performed over several $100 \mu \mathrm{CT}$-slices. The use of the metallic filter improves the determination of the absolute amount of formed bone, which is, however, still questionable.

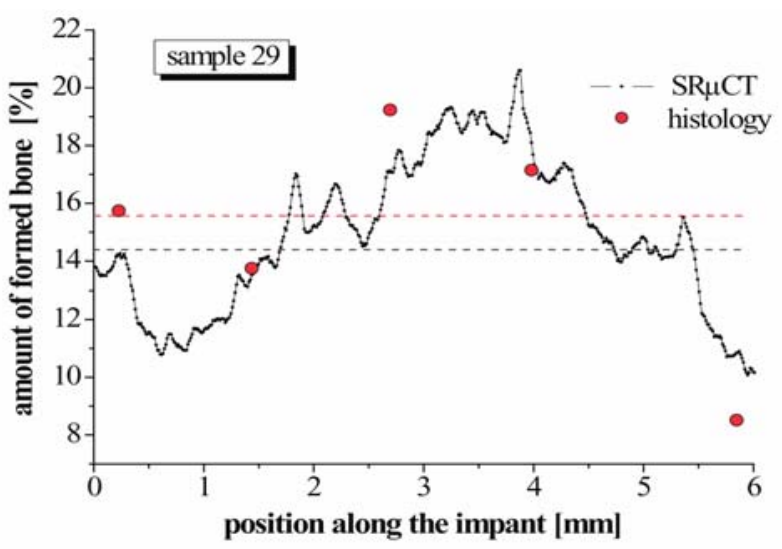

Figure 7. The bone formation along the implant axis is not constant. It varies by a factor of 2 . The small dots represent the SR $\mu \mathrm{CT}$ data, whereby the bright, red dots correspond to the analysis of the classical histology. Note the reasonable agreement between both techniques, especially for the average values given by the dashed lines in red for classical histology and in black for $\mathrm{SR} \mu \mathrm{CT}$. $\mathrm{SR} \mu \mathrm{CT}$, however, provides much more data, which give rise to the clear, quantitative impression, how the bone formation can vary along the implant axis.
The bone amount detected in each slice along the symmetry axis of the implant (Sample 29) is represented in Figure 7 . The big dots in the diagram correspond to the results from the histological images, in reasonable agreement with $\mathrm{SR} \mu \mathrm{CT}$ (small connected dots).

Comparing the amount of bone detected inside the ROI, one finds significant differences for the various modified implant surfaces investigated as demonstrated in Figure 8 . The results obtained by classical histology and SR $\mu \mathrm{CT}$ are generally equivalent for the different surface modifications. Single mean values, however, disagree. For example, for Sample 30 the relative difference between $\mathrm{SR} \mu \mathrm{CT}$ and classical histology is as large as $44 \%$. Due to the very limited number of slices used in classical histology the value may be misleading, as indicated by the error bar, which is too small $(0.7 \%)$ compared with the rather typical value of Sample 29, which is $10.7 \%$. This effect may become clearer in Figure 7, which shows the data scattering along the implant.

Figure 9a is the result of the described integration of bone values along the implant axis, which were found around implants with RGD-peptide immobilisation (Sample 29). The comparison of detected bone amount for $\mathrm{SR} \mu \mathrm{CT}$, microfocus-CT and histological images for this sample as the function of the implant distance is shown in Figure $9 \mathrm{~b}$. Using microfocus-CT, the strong artefacts and especially the beam hardening effects result in the detection of higher absorbing species, not present in reality, up to a distance of about $200 \mu \mathrm{m}$ to the implant surface. At the distance of about $200 \mu \mathrm{m}$ the results of the different methods for the bone detection are identical. Comparing the results of classical histology and $\mathrm{SR} \mu \mathrm{CT}$, there exists only a difference of about $10 \%$ at the interface, which may be caused by the partial volume effect. The increase of detected bone at distances between $250 \mu \mathrm{m}$ to $500 \mu \mathrm{m}$ from the implant surface for the histological images was not found using the SR $\mu \mathrm{CT}$ and is attributed, on the one hand, to the diffuse mineralising fibrosis, not fully suppressed in the segmentation of the von Kossa stained slices and, on the other hand, to the choice of the limited number of histological slices.

\section{Discussion}

This comprehensive study on the visualization and quantification of bone formation around titanium implants using $\mu \mathrm{CT}$ demonstrates the huge differences of tomograms from microfocus- and synchrotron X-ray sources (cf. Fig. 3 ). Only the visualization of the morphology of mineralised bone using SR $\mu \mathrm{CT}$ shows a reasonable agreement of the bone morphology with the classical histological micrographs.

A main problem of microfocus-CT investigations of highly absorbing materials (e.g., titanium) is their energydependent absorption. The broad spectrum of energies causes strong artefacts at the implant surface as seen in the reconstruction from microfocus-CT-systems, termed beam hardening (Fig. 3b). These artefacts are especially pronounced for the given cuboid-shape implant geometry. The metallic pre-filter with the thickness between 0.05 and $0.2 \mathrm{~mm}$ preferentially absorbs the low-energy tail of the $\mathrm{X}$-ray spectrum and can, therefore, reduce the artefacts. Therefore, the $\mu \mathrm{CT}$-system of the BAM provided better results than the one of TUD, where no metallic filter has 


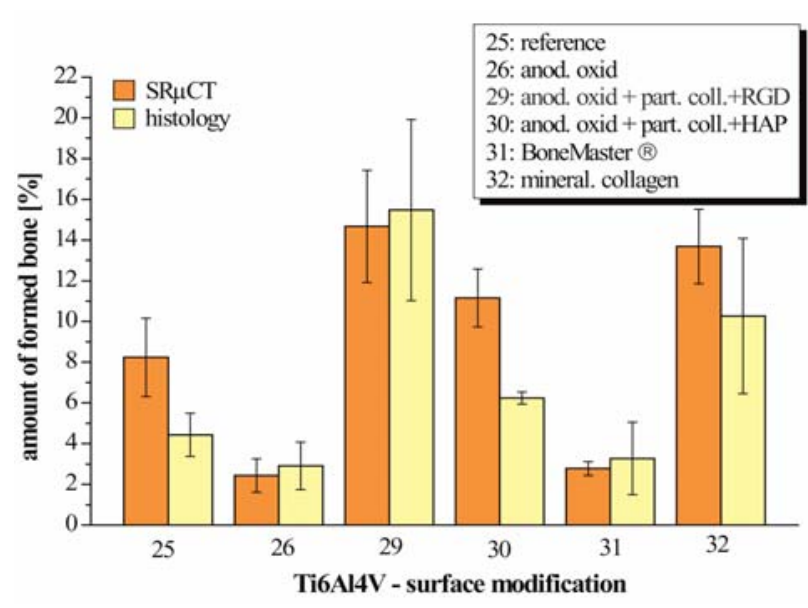

Figure 8. The statistical analysis of bone formation around the different, surface-modified titanium implants clearly shows that, in general, $\mathrm{SR} \mu \mathrm{CT}$ and classical histology lead to very similar results. The $\mathrm{SR} \mu \mathrm{CT}$ data including the error bars, however, are much more reliable than the results of the classical histology, since several hundreds of slices without any preparation artefact are incorporated into the analysis. The limited number of rather randomly chosen slice positions for the classical histology and the use of the von Kossa stain for the Samples 25-26 and 30-32 can explain the differences of the results for the Samples 25 and 30.

been used (cp. Fig. 6). But, in particular, at the titanium/ bone interface the effect is still dominating (Rao and Alfidi, 1981). Furthermore, the filtering leads to the eminent reduction of the total photon flux. As the flux and digital resolution are proportional to each other (Müller et al., 1985), the use of the filter limits the detection accuracy.

Synchrotron radiation sources produce parallel X-rays of high intensity. Although the double crystal monochromator drastically reduces the photon flux, the flux is still comparable with the flux of microfocus X-ray sources. In addition, the parallel beam geometry allows the slice-by-slice reconstruction of the tomogram. SR $\mu \mathrm{CT}$ with synchrotron radiation sources of the second generation as used for the present study leads to histograms of the local absorption coefficients that have exactly the Gaussian shape even for multi-component samples (cf. Fig. 3a). Consequently, it is rather simple to determine the threshold for the segmentation of different components such as mineralised bone and embedding material, even if the related peaks significantly overlap. The tomograms from microfocus-CT, however, provide histograms that deviate more or less from this Gaussian shape. Although median filters can help to improve the segmentation of different components within the tomogram, they cause changes in the shape of the peaks in the histograms, which may complicate the quantitative analysis. It is supposed that a replacement of PMMA embedding by materials with absorption values differing from those of bone will improve the automatic segmentation of bone in the $\mu \mathrm{CT}$ tomograms. Here, we have just used the PMMA well introduced in classical histology.

The segmentation of bony tissue in histological im- (a)

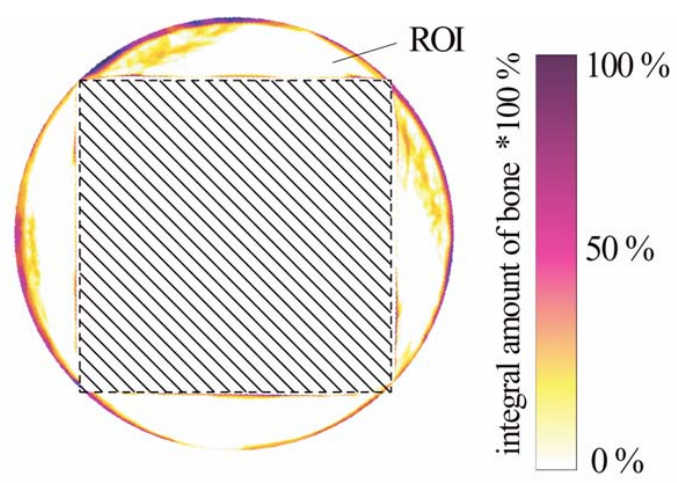

(b)

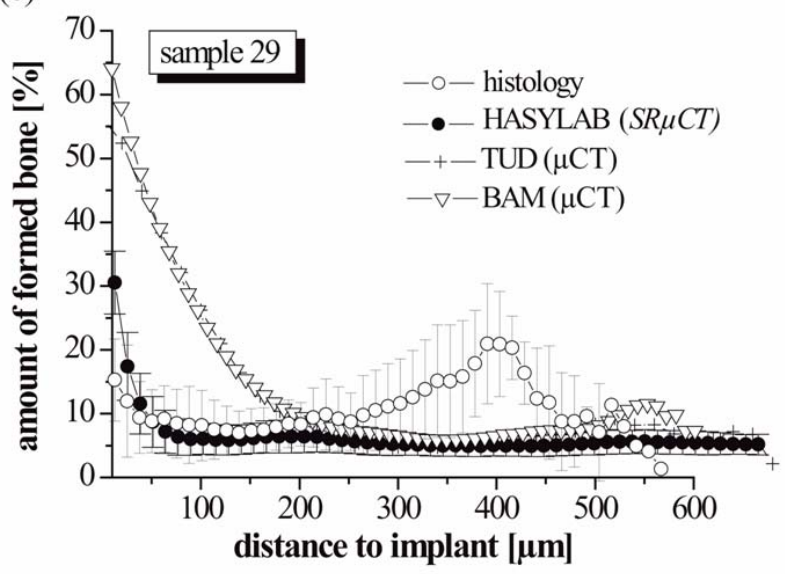

Figure 9. The image (a) shows the average slice generated by the integration of the normalised individual slices for Sample 29. It clearly verifies that the bone predominately forms at the implant edges but the amount can differ from edge to edge. The analysis of the mineralised bone (b) according to Figure $2 \mathrm{~d}$ shows again that the tomograms of microfocus- $\mu \mathrm{CT}$ systems results in misleading values up to distances of $200 \mu \mathrm{m}$ to the implant surface. Classical histology and SR $\mu \mathrm{CT}$ give rise to almost identical behaviour. Just the pixels directly at the interface between bone and implant show partial volume effects for SR $\mu \mathrm{CT}$. The peak in the data of classical histology at around $400 \mu \mathrm{m}$ to the implant surface is attributed to the diffuse mineralising fibrosis, detected in the von Kossa stained slices but not in the $\mu \mathrm{CT}$-data and to the choice of the histological sections.

ages is sometimes difficult since the stained bone can exhibit the similar colour as the implant in the micrograph. Here, the reliable segmentation of bone from the embedding material and the implant was only possible in the methylene-blue stained micrographs. The manual segmentation of bone at the implant surface gives rise to errors if titanium particles from the preparation procedures are present in bony tissue and embedding material or the bony tissue is detached from the implant.

The SR $\mu$ CT-slices along the implant axis reveal the significant variation of bony tissue in the ROI (cf. Fig. 7). The corresponding histological data show reasonable agreement with these values. The variation of the bone formation along the implant axis indicates the strong dependence of the mean value on the limited number of ran- 
domly chosen cutting positions in classical histology. If, for the histology, the slices selected along the implant axis have a distance, which is large in comparison with the changes of newly formed bone, the quantification of bone formation can become questionable. As demonstrated in Figure $9 \mathrm{~b}$, a significant difference can be seen in the amount of bone detected by histology compared to SR $\mu \mathrm{CT}$ at implant distances of 250 to $450 \mu \mathrm{m}$, which cannot fully be explained by the insufficient segmentation of the von Kossa stained bony tissue. The automatic image analysis of hundreds of (virtual) SR $\mu$ CT-slices along the implant axis can master these problems of classical histological studies.

Although $\mu \mathrm{CT}$ is a superior non-destructive technique it has several constraints. First, the scintillator crystals naturally have defects, increasing with lifetime, that result in bright or dark spots on the projections, which result in ring-like artefacts in the reconstructed $3 \mathrm{D}$-data. There already exist several routines to eliminate these artefacts before and after reconstruction (Tang et al., 2001), but the quantitative data evaluation is often affected. A second important limitation is the spatial resolution of the detector (without complicated X-ray optics), which includes the restricted size of the pixels. Structures with a size of the pixel length or smaller cannot be detected in an appropriate manner. The camera just records a mean value of each pixel with statistical noise (Chesler et al., 1977). Consequently, we always find partial volume effects at interfaces, as seen in the diagram in Figure 9b. The partial volume effects also shift the centre of the peaks in the histograms towards the mean value. This means that the partial volume effects modify the Gaussian shape of the peaks. A third problem, when investigating materials with X-rays is energy-dependent absorption. Using conventional sources this effect results in beam hardening, i.e. areas at the outer shape of the sample show an apparently stronger absorption. Consequently, it is important for $\mu \mathrm{CT}$ to apply $\mathrm{X}$-ray beams of photons with almost constant energy. The energy-dependent absorption, however, is problematic even with monochromatic X-rays since the energy cannot be adapted to the different materials of the embedded explant. Thus strongly absorbing components can exhibit star-like features in the tomogram. The dual-energy method in SR $\mu$ CT (Dilmanian et al., 1997) can help to reduce the problem. Although the interactions of X-rays with condensed matter are rather moderate, refraction effects are observed at internal and external interfaces. Since the refractive index of X-rays is close to unity, the energy of the $\mathrm{X}$-rays stays almost constant. But because the tangential component of the wave vector has to be conserved at the interface, a result of the Maxwell equations, the X-rays can change their direction considerably, when the beam hits the interface at oblique incidence. The phenomenon can even lead to total reflection. This refraction effect can be quantified similar to electron beam refraction (Müller et al., 1997). It is visible in the slices of Figure 3. In the extension of the interface the intensity is reduced, an effect more pronounced for the conventional $\mu \mathrm{CT}$ due to the white beam. Using synchrotron radiation the effect is drastically reduced but still present. It is unclear, how far the artefacts can be reduced by the incorporation of refraction into the reconstruction algorithm. The authors are not aware of such a procedure.

The used animal model caused some difficulties when distinguishing between newly formed and already existing hard tissue near the outer ROI border. Thus a different animal model with cylindrical implants and defined cavities is currently examined. With a high number of identical samples and the use of similar analysis procedures, the promising results of peri-implant bone analysis with $\mathrm{SR} \mu \mathrm{CT}$ in accordance with histological findings will be consolidated in a forthcoming publication.

\section{Acknowledgements}

The financial support from Deutsche Forschungsgemeinschaft (WI 1897/1, WO 494/10), from Schweizerischer Nationalfonds (2153-057127.99) and from HASYLAB (II-99-077) is gratefully acknowledged.

\section{References}

Becker D, Geissler U, Hempel U, Bierbaum S, Scharnweber D, Worch H, Wenzel KW (2002) Proliferation and differentiation of rat calvarial osteoblasts on type I collagen-coated titanium alloy. J Biomed Mater Res 59: 516-527.

Becker J, Meisser T, Neukam FW, Knöpfler W, Graf H-L, Reichart P (1991) Tierexperimentelle Untersuchungen zur Einheilung von ANOF-beschichteten Titanimplantaten (Investigation concerning the osseointegration of ANOF coated titanium implants in animal experiments). Zeitschrift für Zahnärztliche Implantologie 7: 162-169.

Beckmann F, Bonse U (2000) Attenuation- and phasecontrast microtomography using synchrotron radiation for the 3-dimensional investigation of specimens consisting of weakly and normally absorbing elements Mat Res Soc Symp Proc 590: 265-271.

Beckmann F, Bonse U, Busch F, Gunnewig O (1997) $\mathrm{X}$-ray microtomography (microCT) using phase contrast for the investigation of organic matter. J Comput Assist Tomogr 21: 539-553.

Chappard D, Aguado E, Hure G, Grizon F, Basle MF (1999) The early remodeling phases around titanium implants: a histomorphometric assessment of bone quality in a 3- and 6-month study in sheep. Int J Oral Maxillofac Implants 14: 189-196.

Chesler DA, Riederer SJ, Pelc NJ (1977) Noise due to photon counting statistics in computed X-ray tomography. J Comput Assist Tomogr 1: 64-74.

Dard M, Sewing A, Meyer J, Verrier S, Roessler S, Scharnweber D (2000) Tools for tissue engineering of mineralized oral structures. Clin Oral Investig 4: 126-129.

Dempster DW, Cosman F, Kurland ES, Zhou H, Nieves J, Woelfert L, Shane E, Plavetic K, Muller R, Bilezikian J, Lindsay R (2001) Effects of daily treatment with parathyroid hormone on bone microarchitecture and turnover in patients with osteoporosis: a paired biopsy study. J Bone Miner Res 16: 1846-1853. 
Dilmanian FA, Wu XY, Parsons EC, Ren B, Kress J, Button TM, Chapman LD, Coderre JA, Giron F, Greenberg D, Krus DJ, Liang Z, Marcovici S, Petersen MJ, Roque CT, Shleifer M, Slatkin DN, Thomlinson WC, Yamamoto K, Zhong Z (1997) Single-and dual-energy CT with monochromatic synchrotron X-rays. Phys Med Biol 42: 371387.

Fartash B, Arvidson K, Ericsson I (1990) Histology of tissues surrounding single crystal sapphire endosseous dental implants: an experimental study in the beagle dog. Clin Oral Implants Res 1: 13-21.

Feldkamp LA, Davis LC, Kress J. W. (1984) Practical cone-beam algorithm. J Opt Soc Amer 1: 612-619.

Giavaresi G, Fini M, Cigada A, Chiesa R, Rondelli G, Rimondini L, Torricelli P, Aldini NN, Giardino R (2003) Mechanical and histomorphometric evaluations of titanium implants with different surface treatments inserted in sheep cortical bone. Biomaterials 24: 1583-1594.

Goebbels J, Weidemann G, Dittrich R, Mangler M, Tomandl G (2002) Functionally graded porosity in ceramics - analysis with high-resolution computed tomography. Proc. Innovative processing and synthesis of ceramics, glasses, and composites V (ed. J. P. Singh), Ceramic Transactions 129: 113-124.

Hallgren C, Sawase T, Ortengren U, Wennerberg A (2001) Histomorphometric and mechanical evaluation of the bone-tissue response to implants prepared with different orientation of surface topography. Clin Implant Dent Relat Res 3: 194-203.

Ivanoff CJ, Hallgren C, Widmark G, Sennerby L, Wennerberg A (2001) Histologic evaluation of the bone integration of $\mathrm{TiO}(2)$ blasted and turned titanium microimplants in humans. Clin Oral Implants Res 12: 128134.

Johnsson AA, Sawaii T, Jacobsson M, Granstrom G, Turesson I (2000) A histomorphometric and biomechanical study of the effect of delayed titanium implant placement in irradiated rabbit bone. Clin Implant Dent Relat Res 2: 42-49.

Kak AC, Slaney M (2001) Principles of Computerized Tomographic Imaging. Society of Industrial and Applied Mathematics, Philadelphia, PA.

Kinney JH, Haupt DL, Balooch M, Ladd AJ, Ryaby JT, Lane NE (2000) Three-dimensional morphometry of the L6 vertebra in the ovariectomized rat model of osteoporosis: biomechanical implications. J Bone Miner Res 15: 1981-1991.

Kron T (1998) Applications of synchrotron radiation X-rays in medicine. Phys Med Biol 43: 215-216.

LeGeros RZ, Craig RG (1993) Strategies to affect bone remodeling: osteointegration. J Bone Miner Res 8 Suppl 2: S583-S596.

Marquardt DW (1969) An algorithm for least-squares estimation of nonlinear parameters. J Soc Industr Appl Math 11: 431-441.

Morton EJ, Webb S, Bateman JE, Clarke LJ, Shelton CG (1990) Three-dimensional x-ray microtomography for medical and biological applications. Phys Med Biol 35: 805-820.

Müller A, Ruegsegger P, Seitz P (1985) Optimal CT settings for bone evaluations. Phys Med Biol 30: 401-409.
Müller B, Beckmann F, Huser M, Maspero F, Szekely G, Ruffieux K, Thurner P, Wintermantel E (2002) Nondestructive three-dimensional evaluation of a polymer sponge by micro-tomography using synchrotron radiation. Biomol Eng 19: 73-78.

Müller B, Henzler H (1997) Comparison of reflection high-energy electron diffraction and low-energy electron diffraction using high-resolution instrumentation. Surf Sci 389: 338-348.

Nöthe M, Pischang K, Ponízil P, Bernhardt R, Kieback B (2002) Analysis of sinter processes by X-ray computer tomography. In: Advances in Powder Metallurgy and Particulate Materials. MPIF, Princeton, NJ. Part 13, pp.176184.

Nuzzo S, Lafage-Proust MH, Martin-Badosa E, Boivin G, Thomas T, Alexandre C, Peyrin F (2002) Synchrotron radiation microtomography allows the analysis of threedimensional microarchitecture and degree of mineralization of human iliac crest biopsy specimens: effects of etidronate treatment. J Bone Miner Res 17: 1372-1382.

Rao PS, Alfidi RJ (1981) The environmental density artifact: a beam-hardening effect in computed tomography. Radiology 141: 223-227.

Roessler S, Scharnweber D, Worch H, Sewing A, Dard M (2001) Biomimetic coatings functionalized with adhesion peptides for dental implants. J Mater Sci: Mater in Med 12: 871-877.

Roessler S, Sewing A, Stolzel M, Born R, Scharnweber D, Dard M, Worch H (2003) Electrochemically assisted deposition of thin calcium phosphate coatings at nearphysiological $\mathrm{pH}$ and temperature. J Biomed Mater Res 64A: 655-663.

Ruegsegger P, Koller B, Müller R (1996) A microtomographic system for the nondestructive evaluation of bone architecture. Calcif Tissue Int 58: 24-29.

Scharnweber D, Beutner R, Roessler S, Worch H (2002) Electrochemical behaviour of titanium based materials - Are there relations to biocompatibility? J Mater Sci: Mater in Med 13: 1215-1220.

Scharnweber D, Born R, Flade K, Roessler S, Stoelzel M, Worch H (2004) Mineralization behaviour of collagen type I immobilized on different substrates. Biomaterials 25: 2371-2380.

Schliephake H, Scharnweber D, Dard M, Robetaler S, Sewing A, Huttmann C (2003) Biological performance of biomimetic calcium phosphate coating of titanium implants in the dog mandible. J Biomed Mater Res 64A: 225-234.

Schliephake H, Scharnweber D, Dard M, Rossler S, Sewing A, Meyer J, Hoogestraat D (2002) Effect of RGD peptide coating of titanium implants on periimplant bone formation in the alveolar crest. An experimental pilot study in dogs. Clin Oral Implants Res 13: 312-319.

Sennerby L, Wennerberg A, Pasop F (2001) A new microtomographic technique for non-invasive evaluation of the bone structure around implants. Clin Oral Implants Res 12: 91-94.

Tang X, Ning R, Yu R, Conover D (2001) Cone beam volume CT image artifacts caused by defective cells in $\mathrm{X}$ ray flat panel imagers and the artifact removal using a wavelet-analysis-based algorithm. Med Phys 28: 812-825.

Truhlar RS, Morris HF, Ochi S (2000) Stability of the 
bone-implant complex. Results of longitudinal testing to 60 months with the Periotest device on endosseous dental implants. Ann Periodontol 5: 42-55.

Turner RT, Francis R, Brown D, Garand J, Hannon KS , Bell NH (1989) The effects of fluoride on bone and implant histomorphometry in growing rats. J Bone Miner Res 4: 477-484.

Van Oosterwyck H, Duyck J, Vander Sloten J, Van der Perre G, Jansen J, Wevers M, Naert I (2000) Use of microfocus computerized tomography as a new technique for characterizing bone tissue around oral implants. J Oral Implantol 26: 5-12.

Wennerberg A, Albrektsson T, Andersson B, Krol JJ (1995) A histomorphometric and removal torque study of screw-shaped titanium implants with three different surface topographies. Clin Oral Implants Res 6: 24-30.

Wigianto R, Ichikawa T, Kanitani H, Kawamoto N, Matsumoto N, Ishizuka H (1999) Three-dimensional bone structure around hydroxyapatite and titanium implants in rabbits. Clin Oral Implants Res 10: 219-225.

Wolf E, Pompe B (1980) Vereinfachte Methacrylateinbettung für unentkalkte Knochenschnitte. (Simplified method of methacrylate embedding for undecalcified bone sections). Zentralbl Allg Pathol 124: 551-556.

\section{Discussion with Reviewers}

J. Gasser: Do authors have any explanation why the preparation artefacts in the histology sections are particularly strong at a distance of 300 to $450 \mu \mathrm{m}$ from the implant? It could have been expected that the largest impact of preparation would occur in the area in close proximity to the implant/bone interface.

Authors: There are two reasons for the difference between classical histology and SR $\mu \mathrm{CT}$ at the distance of about $400 \mu \mathrm{m}$. First, the difference is the direct consequence of the limited number of histological slices rather randomly chosen. Second, as the result of the staining and the subsequent automatic segmentation the amount of bone is often higher for classical histology than for $\mathrm{SR} \mu \mathrm{CT}$. This phenomenon is attributed to the staining of partly mineralized bone. In SR $\mu \mathrm{CT}$ this kind of bone is suppressed, whereas in classical histology it is acquired.
J. Kirkpatrick: In the discussion the authors rightly refer to the difficulties in clearly identifying newly formed bone. They also suggest that a new animal model is required. How exactly do the authors propose to distinguish between preexisting bone and newly formed bone around their implants?

Authors: First, it is known that newly formed bone exhibits the lower X-ray absorption than preexisting bone, because it can take months before the osteons mature. The absorption directly reflects the degree of mineralization. A detailed study on the quantification of the degree of mineralization of bone using $\mathrm{SR} \mu \mathrm{CT}$ is given for example by Nuzzo et al. (2002). Second, one could incorporate appropriate agents to stain the newly formed bone. This route, however, complicates the procedure.

B. Rahn: How precise is the synchrotron method in assessing osteointegration, i.e. determination of direct bone deposition without any non-mineralised layers between bone and implant? Is it still true that the last 50 micrometers, as it may be concluded from Figure 9, are reserved for conventional histology, and the last micrometer for electron microscopy?

Authors: For the selected implant size and geometry and the presently available data reconstruction procedures the precision of SR $\mu \mathrm{CT}$ in assessing osteointegration is only several $10 \mu \mathrm{m}$. Please note that, as the result of the preparation, the data of classical histology and electron microscopy have to be also handled with care. Therefore, it is highly desirable to further develop quantitative $\mathrm{SR} \mu \mathrm{CT}$. First, one can adopt the implant size and geometry to the $\mathrm{SR} \mu \mathrm{CT}$ method, reducing the implant diameter and using rounded shapes. Second, one might incorporate the interface scattering into the reconstruction algorithm in a recursive manner. These measures should improve the spatial resolution of $\mathrm{SR} \mu \mathrm{CT}$ at the interface between bone and titanium implant towards $1 \mu \mathrm{m}$. However we want to point out again that classical histology and SR $\mu \mathrm{CT}$ of implants with surrounding bony tissue are rather complementary than competing techniques.

\section{Additional Reference}

Nuzzo S, Peyrin F, Cloetens P, Baruchel J, Boivin G (2002) Quantification of the degree of mineralization of bone in three dimensions using synchrotron radiation microtomography. Med Phys 29: 2672-2681. 